\title{
Advances in hybrid finite element - boundary integral - multilevel fast multipole - uniform geometrical theory of diffraction method
}

\author{
A. Tzoulis ${ }^{1}$ and T. F. Eibert $^{2}$ \\ ${ }^{1}$ FGAN-FHR, Neuenahrer Str. 20, 53343 Wachtberg, Germany \\ ${ }^{2}$ Institute of Radio Frequency Technology, Universität Stuttgart, 70550 Stuttgart, Germany
}

\begin{abstract}
Numerical modeling of problems including composite metallic/dielectric objects with arbitrary shapes and electrically large conducting objects within a common environment is performed in an optimum way with the recently developed powerful hybrid numerical method, which combines the Finite Element Boundary Integral (FEBI) method and the Multilevel Fast Multipole Method (MLFMM) with the Uniform Geometrical Theory of Diffraction (UTD), giving full electromagnetic coupling between all involved objects. In this contribution, the hybrid FEBI-MLFMM-UTD method is extended to double diffracted fields on pairs of straight metallic edges, formulated with the hard and soft scalar diffraction coefficients of UTD. The diffraction points on each pair of edges are determined by an iterative three-dimensional parametric realization of the generalized Fermat's principle. The divergence factor of the double diffracted field is computed by multiplying the appropriate divergence factors of the single diffracted UTD fields on each edge for the particular case. Thereby, the ray caustic distance of the diffracted field at the second edge is determined by linear interpolation between the radii of curvature in the two principal planes of the incident astigmatic ray tube. Further, fast near-field computation in the postprocessing stage of the hybrid method is extended in each translation domain to ray optical contributions due to the presence of electrically large objects, according to the hybridization of MLFMM with UTD. Formulations and numerical results will be presented.
\end{abstract}

Correspondence to: A. Tzoulis

(tzoulis@fgan.de)

\section{Introduction}

Recently, a powerful hybrid numerical method was introduced, which combines the Finite Element Boundary Integral (FEBI) method and the Multilevel Fast Multipole Method (MLFMM) with the Uniform Geometrical Theory of Diffraction (UTD), giving full electromagnetic coupling between composite metallic/dielectric objects with arbitrary shapes and electrically large conducting objects within a common environment (Tzoulis, Oct. 2005). In that work, single and multiple reflections on flat metallic objects were considered, whereas diffractions were taken into account only by single mechanisms on straight metallic edges.

In this contribution, the hybrid FEBI-MLFMM-UTD method is extended to double diffracted fields on pairs of straight metallic edges, formulated with the scalar hard and soft diffraction coefficients of UTD according to (Kouyoumjian, 1974). For configurations, in which the second diffraction point is in the transition region of the first, the scalar diffraction coefficients of UTD are not valid, because the incident field on the second edge is not purely ray optical, by means of rapid spatial variations due to the Fresnel transition functions in the diffraction coefficients of the first edge. Double diffracted field formulations, which are valid in transition regions of the first edge, have been developed in (Tiberio et al., 1989)-(Albani, 2005) using several approaches, such as a spectral extension of the UTD or spherical spectral synthesis.

In the present implementation of standard double diffracted UTD fields, the diffraction points on the pair of edges are determined by an iterative three-dimensional parametric realization of the generalized Fermat's principle, allowing treatment of non-coplanar and skewed edges. According to this, the position vectors of the diffraction points are expressed in terms of two parameters defined on the edges. On each edge, the diffraction point is computed in closed form for a given set of source and observation point,

Published by Copernicus Publications on behalf of the URSI Landesausschuss in der Bundesrepublik Deutschland e.V. 


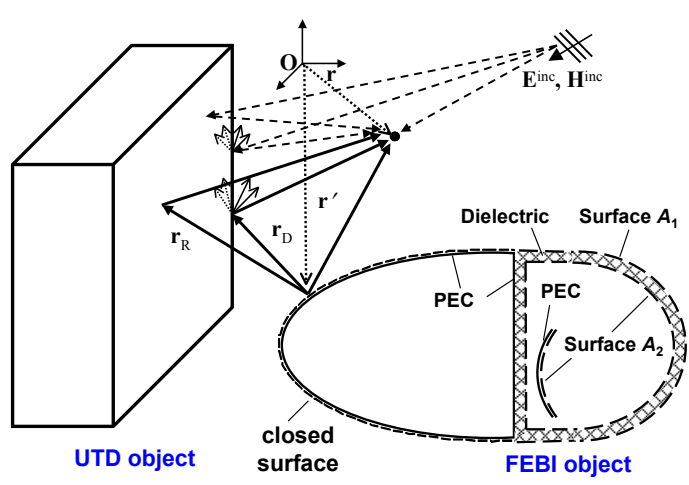

Fig. 1. Hybrid FEBI-MLFMM-UTD configuration.

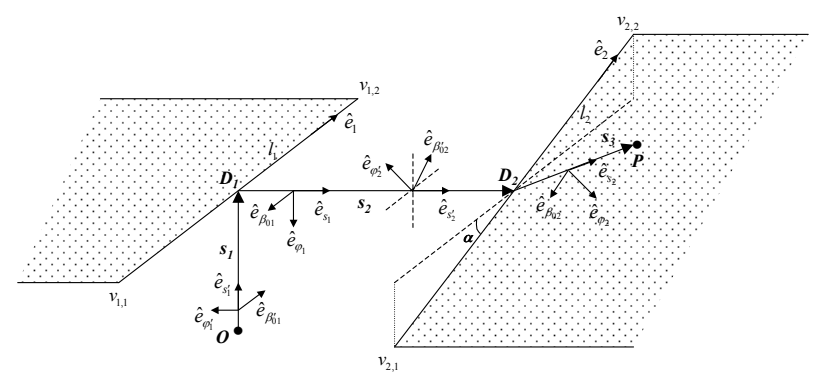

Fig. 2. Double diffraction edge configuration.

where the corresponding parameter is determined by a threedimensional realization of the generalized Fermat's principle. For double diffraction, the above procedure is repeated iteratively for each edge, using each time a point on the other edge as source or observation point, respectively, until the law of diffraction is satisfied on both edges with desired accuracy (Ivrissimtzis, 1991).

The divergence factor of the double diffracted field is determined by multiplying the appropriate divergence factors of the single diffracted UTD fields on each edge for the particular case. Thereby, the ray caustic distance of each single diffracted field is needed and for straight metallic edges it is given by the radius of curvature of the incident wavefront in the plane of incidence. For the diffracted field at the second edge, the incident wave is an astigmatic ray tube and the ray caustic distance is determined by linear interpolation between the radii of curvature in the two principal planes of the incident astigmatic ray tube.

Further, near-field computations in the postprocessing stage of the hybrid method are accelerated by MLFMM according to (Tzoulis, 2005), in which high-frequency contributions due to the presence of electrically large objects are taken into account within each translation domain according to the hybridization of MLFMM with UTD introduced in (Tzoulis, Oct. 2005). In the following, formulations will be given and numerical results will be shown.

\section{Formulation}

The configuration for the hybrid FEBI-MLFMM-UTD concept can be seen in Fig. 1. Composite metallic/dielectric objects are treated with the FEBI technique, in which fast integral equation solution is achieved by MLFMM. Electrically large metallic objects, which are in the same environment, are treated with UTD and full coupling with the surface currents of the Boundary Integral (BI) part is given according to the hybridization introduced in (Tzoulis, Oct. 2005). In that work, single and multiple reflections on flat metallic objects were considered, whereas diffractions were taken into account only by single mechanisms on straight metallic edges.

In the hybrid method, double diffracted UTD field contributions on pairs of edges are taken into account according to the configuration shown in Fig. 2. The edges of the pair can be in general non-coplanar and skewed by an angle $\alpha$. The diffraction points $D_{1}$ on edge 1 and $D_{2}$ on edge 2 are numerically determined by an iterative three-dimensional parametric realization of the generalized Fermat's principle. According to this, the position vectors of the diffraction points are expressed in terms of two parameters defined on the edges as

$\mathbf{r}_{D_{j}}=\mathbf{r}_{v_{j, 1}}+p_{j} l_{j} \hat{e}_{j}, \quad j=1,2$

where $\mathbf{r}_{v_{j, 1}}$ and $l_{j}$ are the position vector of the first vertex and the length of edge $j$, respectively. The parameter $p_{j}$ is defined on edge $j$ and takes values between $[0,1]$. Also, $\hat{e}_{j}$ is the unit vector along edge $j$ defined from the first to the second vertex of the edge. On each edge $j$, the diffraction point $\mathbf{r}_{D_{j}}$ is computed in closed form for a given set of source and observation point, by a three-dimensional realization of the generalized Fermat's principle, as shown in Fig. 3. According to this, the parameter $p_{j}$ is given by

$p_{j}=\frac{x_{d}+x_{1}}{l_{j}}$,

with

$x_{d}=\frac{h_{1}}{h_{1}+h_{2}}\left(x_{2}-x_{1}\right)$.

$h_{1}$ and $h_{2}$ are the distances of the source and observation point from the edge, respectively. Also, $x_{1}$ and $x_{2}$ are the distances of the projection of the source and observation point from the first vertex of the edge, respectively. For double diffraction, the above procedure is repeated iteratively for each edge, using each time a point on the other edge as source or observation point, respectively, until the law of diffraction is satisfied on both edges with desired accuracy (Ivrissimtzis, 1991). This is typically achieved after only few iterations.

The double diffracted field at the observation point $P$ has the form

$\mathbf{E}^{d d}(P)=\overline{\mathbf{D}}_{2} \cdot \overline{\mathbf{D}}_{1} \cdot \mathbf{E}^{i}\left(D_{1}\right) A_{1} A_{2} \mathrm{e}^{-j k\left(s_{2}+s_{3}\right)}$, 


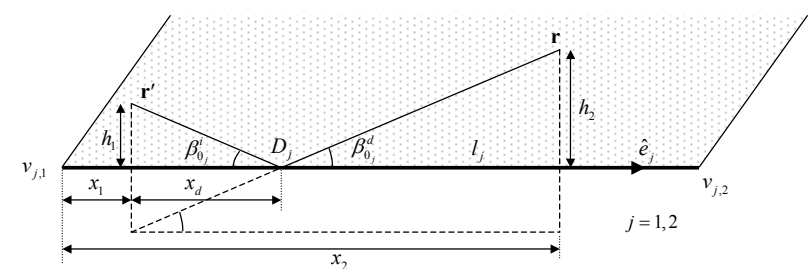

Fig. 3. Computation of diffraction point on each edge.

where $\mathbf{E}^{i}\left(D_{1}\right)$ is the incident field at $D_{1}$ and

$\overline{\mathbf{D}}_{j}=-D_{\|_{j}} \hat{e}_{\beta_{0 j}} \hat{e}_{\beta_{0 j}^{\prime}}-D_{\perp_{j}} \hat{e}_{\varphi_{j}} \hat{e}_{\varphi_{j}^{\prime}}, \quad j=1,2$

are the diffraction tensors, in which $\hat{e}_{\beta_{01,2}^{\prime}} \hat{e}_{\varphi_{1,2}^{\prime}}$ and $\hat{e}_{\beta_{01,2},} \hat{e}_{\varphi_{1,2}}$ are the transversal components in the edge-fixed coordinate system of incidence and diffraction on edge 1 or edge 2 , respectively, as shown in Fig. 2. The double diffracted field is formulated with the scalar diffraction coefficients $D_{\|}, D_{\perp}$ of UTD (Kouyoumjian, 1974). In addition,

$A_{1}=\sqrt{\frac{s_{1}}{s_{2}\left(s_{2}+s_{1}\right)}}$

is the divergence factor of the diffracted field at edge 1 . In that, the ray caustic distance $\rho_{e_{1}}$ is assumed to be equal to $s_{1}$, which stands for the case of incident spherical wavefront on a straight edge. Further,

$A_{2}=\sqrt{\frac{\rho_{e_{2}}}{s_{3}\left(s_{3}+\rho_{e_{2}}\right)}}$

is the divergence factor of the diffracted field at edge 2 . The incident field on edge 2 is the diffracted field at edge 1, which is an astigmatic ray field, as shown in Fig. 4.

The ray caustic distance $\rho_{e_{2}}$ of the diffracted field at edge 2 is determined from the radius of curvature of the incident field in the plane of incidence, defined by $\hat{e}_{s_{2}^{\prime}}$ and $\hat{e}_{2}$. In case of non-coplanar and skewed edges, the plane of incidence at edge 2 is in general somewhere in between the two principal planes of the incident astigmatic ray tube. Consequently, the ray caustic distance $\rho_{e_{2}}$ depends on the radii of curvature $\rho_{e_{21}}^{i}, \rho_{e_{22}}^{i}$ of the incident wavefront in both principal planes, which correspond to the first and second caustic of the incident ray field at edge 2 , respectively. It is obvious, that for coplanar edges $(\alpha=0)$, the plane of incidence at edge 2 coincides with the principal plane corresponding to the second caustic of the incident ray tube and for perpendicular edges $\left(\alpha=\frac{\pi}{2}\right)$, the plane of incidence at edge 2 coincides with the principal plane corresponding to the first caustic of the incident ray tube, which lies on edge 1 . The ray caustic distance $\rho_{e_{2}}$ for values of $\alpha$ between 0 and $\frac{\pi}{2}$ is determined by linear interpolation between these two principal values of $\rho_{e_{2}}$, resulting into

$\rho_{e_{2}}=s_{1}+s_{2}-\frac{2 \alpha}{\pi} s_{1}$,

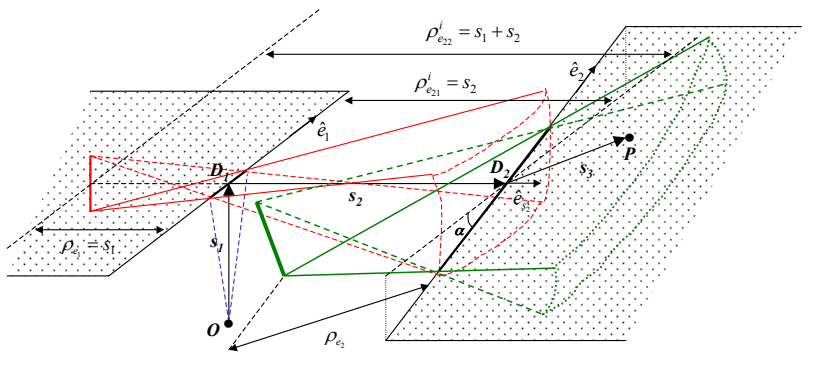

Fig. 4. Ray tubes of double diffraction.

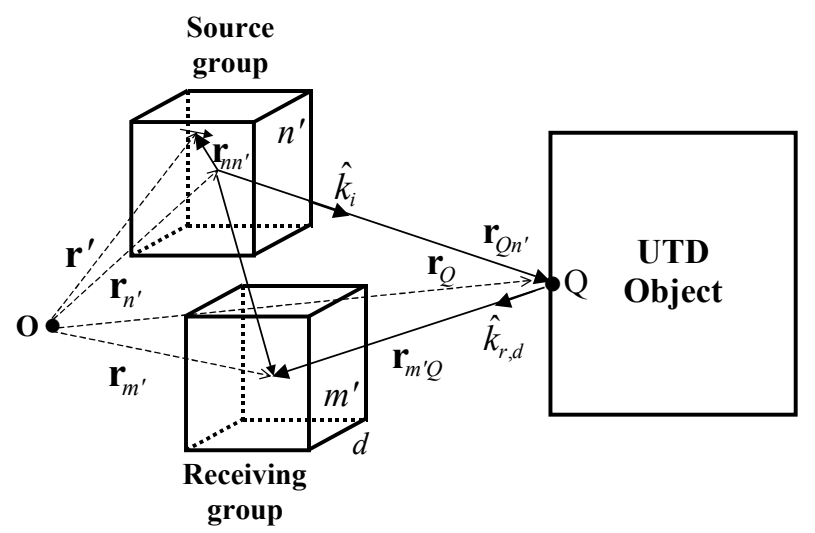

Fig. 5. Hybrid MLFMM-UTD configuration.

where $\alpha$ is given in radians. Consequently, the divergence factor of the diffracted field at edge 2 is given by

$A_{2}=\sqrt{\frac{s_{1}+s_{2}-\frac{2 \alpha}{\pi} s_{1}}{s_{3}\left(s_{3}+s_{1}+s_{2}-\frac{2 \alpha}{\pi} s_{1}\right)}}$.

Finally, near-field computations in the postprocessing stage of the hybrid method are accelerated by the MLFMM approach presented in (Tzoulis, 2005), where additional ray optical contributions due to the presence of electrically large objects are taken into account in both translation domains according to the hybridization of MLFMM with UTD introduced in (Tzoulis, Oct. 2005). The total electric field at an observation point $\mathbf{r}$ in the near-field region of a perfectly conducting arbitrarily shaped object, which is in the same environment with electrically large objects, is computed in the postprocessing stage by

$\mathbf{E}_{t o t}(\mathbf{r})=\mathbf{E}(\mathbf{r})+\mathbf{E}_{U T D}(\mathbf{r})$,

where $\mathbf{E}(\mathbf{r})$ is the direct field contribution given in (Tzoulis, $2005)$ and $\mathbf{E}_{U T D}(\mathbf{r})$ are the ray optical field contributions received at the observation point due to the presence of the electrically large objects. For field points located inside the domain covered by MLFMM groups, the received ray optical contributions due to the presence of the UTD objects are 


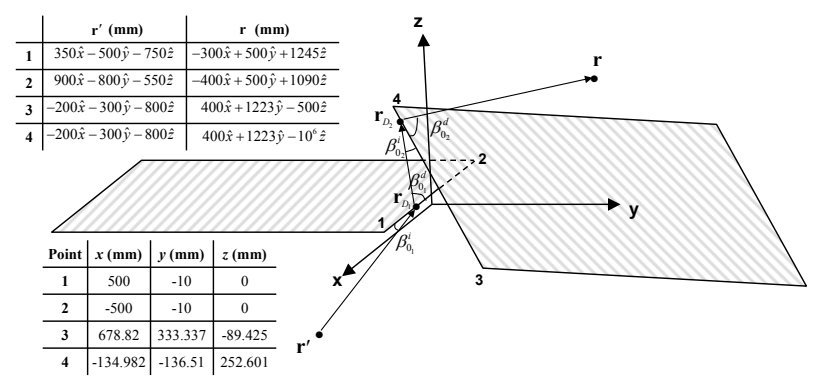

Fig. 6. General example of edges skewed in all directions.

Table 1. Double diffraction points for edge configuration and source/observation points shown in Fig. 6.

\begin{tabular}{ccc}
\hline & $\mathbf{r}_{D_{1}}(\mathrm{~mm})$ & $\mathbf{r}_{D_{2}}(\mathrm{~mm})$ \\
\hline $\mathbf{r}_{1}^{\prime}, \mathbf{r}_{1}$ & $165.0 \hat{x}-10 \hat{y}+0.0 \hat{z}$ & $135.7 \hat{x}+19.8 \hat{y}+138.8 \hat{z}$ \\
$\mathbf{r}_{2}^{\prime}, \mathbf{r}_{2}$ & $279.8 \hat{x}-10 \hat{y}+0.0 \hat{z}$ & $195.6 \hat{x}+54.4 \hat{y}+113.7 \hat{z}$ \\
$\mathbf{r}_{3}^{\prime}, \mathbf{r}_{3}$ & $241.1 \hat{x}-10 \hat{y}+0.0 \hat{z}$ & $318.2 \hat{x}+125.1 \hat{y}+62.1 \hat{z}$ \\
$\mathbf{r}_{4}^{\prime}, \mathbf{r}_{4}$ & $167.2 \hat{x}-10 \hat{y}+0.0 \hat{z}$ & $223.3 \hat{x}+70.4 \hat{y}+10.2 \hat{z}$ \\
\hline
\end{tabular}

given by

$$
\begin{gathered}
\mathbf{E}_{U T D}^{N F}(\mathbf{r})= \\
-j \frac{\omega \mu}{4 \pi} \mathrm{e}^{-j \mathbf{k}_{r} \cdot \mathbf{r}_{m m^{\prime}}} \sum_{s} A_{R_{s}} \overline{\mathbf{R}}_{s}^{E} T_{L}^{U T D}\left(k r_{Q n^{\prime}}\right) \\
\cdot\left(\overline{\mathbf{I}}-\hat{k}_{r} \hat{k}_{i}\right) \cdot \sum_{n} J_{n} \tilde{\boldsymbol{\beta}}_{n}\left(\hat{k}_{i}\right) \\
-j \frac{\omega \mu}{4 \pi} \mathrm{e}^{-j \mathbf{k}_{d} \cdot \mathbf{r}_{m m^{\prime}}} \sum_{v} A_{D_{v}} \overline{\mathbf{D}}_{v}^{E} T_{L}^{U T D}\left(k r_{Q n^{\prime}}\right) \\
\cdot\left(\overline{\mathbf{I}}-\hat{k}_{d} \hat{k}_{i}\right) \cdot \sum_{n} J_{n} \tilde{\boldsymbol{\beta}}_{n}\left(\hat{k}_{i}\right)-\cdots \\
+\mathbf{E}_{U T D}^{i n c}(\mathbf{r}),
\end{gathered}
$$

where

$$
\tilde{\mathbf{J}}_{n}(\hat{k})=J_{n} \tilde{\boldsymbol{\beta}}_{n}(\hat{k})=J_{n} \iint_{A} \boldsymbol{\beta}_{n}\left(\mathbf{r}_{n}\right) \mathrm{e}^{j \mathbf{k} \cdot \mathbf{r}_{n n^{\prime}}} d a^{\prime}
$$

is the $\hat{k}$-space representation of the electric current densities on the surface elements with $\mathbf{k}=k \hat{k}$. The distance vectors $\mathbf{r}_{n n^{\prime}}$ and $\mathbf{r}_{m m^{\prime}}$, as well as the distance $r_{Q n^{\prime}}$ can be seen in Fig. 5. $T_{L}^{U T D}\left(k r_{Q n^{\prime}}\right)$ is the far-field MLFMM translation operator used to translate outgoing ray optical fields from source groups to incoming ray optical fields at receiving groups (Tzoulis, Oct. 2005). $A_{R_{s}}$ and $A_{D_{v}}$ are the divergence and phase factors for reflection and diffraction, respectively, and $\overline{\mathbf{R}}_{s}^{E}$ and $\overline{\mathbf{D}}_{v}^{E}$ the dyadic reflection and diffraction coefficients for electric field defined by basic GO and UTD concepts (Kouyoumjian, 1974). For observation points outside
Table 2. Angles of incidence and diffraction for configuration shown in Fig. 6, using the computed diffraction points of Table 1.

\begin{tabular}{lcccc}
\hline & $\beta_{0_{1}}^{i}\left({ }^{\circ}\right)$ & $\beta_{0_{1}}^{d}\left({ }^{\circ}\right)$ & $\beta_{0_{2}}^{i}\left({ }^{\circ}\right)$ & $\beta_{0_{2}}^{d}\left({ }^{\circ}\right)$ \\
\hline $\mathbf{r}_{1}^{\prime}, \mathbf{r}_{1}$ & 78.33 & 78.33 & 66.69 & 66.69 \\
$\mathbf{r}_{2}^{\prime}, \mathbf{r}_{2}$ & 57.20 & 57.20 & 60.24 & 60.24 \\
$\mathbf{r}_{3}^{\prime}, \mathbf{r}_{3}$ & 117.40 & 117.40 & 128.80 & 128.80 \\
$\mathbf{r}_{4}^{\prime}, \mathbf{r}_{4}$ & 113.35 & 113.35 & 110.04 & 110.04 \\
\hline
\end{tabular}

the MLFMM grouping domain, the ray optical field contributions become

$$
\begin{gathered}
\mathbf{E}_{U T D}^{F F}(\mathbf{r})= \\
-j \frac{\omega \mu}{4 \pi} \sum_{s} A_{R_{s}} \overline{\mathbf{R}}_{s}^{E} T_{L}^{U T D}\left(k r_{Q n^{\prime}}\right) \\
\cdot\left(\overline{\mathbf{I}}-\hat{k}_{r} \hat{k}_{i}\right) \cdot \sum_{n} J_{n} \tilde{\boldsymbol{\beta}}_{n}\left(\hat{k}_{i}\right) \\
-j \frac{\omega \mu}{4 \pi} \sum_{v} A_{D_{v}} \overline{\mathbf{D}}_{v}^{E} T_{L}^{U T D}\left(k r_{Q n^{\prime}}\right) \\
\cdot\left(\overline{\mathbf{I}}-\hat{k}_{d} \hat{k}_{i}\right) \cdot \sum_{n} J_{n} \tilde{\boldsymbol{\beta}}_{n}\left(\hat{k}_{i}\right)-\cdots \\
+\mathbf{E}_{U T D}^{i n c}(\mathbf{r}),
\end{gathered}
$$

where

$$
\begin{aligned}
\mathbf{E}_{U T D}^{i n c}(\mathbf{r}) & =\sum_{s} A_{R_{s}} \overline{\mathbf{R}}_{s}^{E} \cdot \mathbf{E}^{i n c}\left(\mathbf{r}_{R_{s}}\right) \\
& +\sum_{v} A_{D_{v}} \overline{\mathbf{D}}_{v}^{E} \cdot \mathbf{E}^{i n c}\left(\mathbf{r}_{D_{v}}\right)+\cdots
\end{aligned}
$$

are the ray optical contributions of the incident electric field received at the observation point $\mathbf{r}$. The field contributions for magnetic currents, as well as the formulations for the magnetic field in the postprocessing stage can be given in the same way.

\section{Numerical examples}

First, the computation of double diffraction points is tested with the general skewed edge configuration shown in Fig. 6. In the same figure, the Cartesian coordinates of the vertices of the edges, as well as the sets of source and observation points for the determination of the double diffraction points can be seen. The computed diffraction points are shown in Table 1 and the particular angles of incidence and diffraction are shown in Table 2. It can be seen, that the double diffraction points are determined correctly, since in each case the law of diffraction is satisfied.

Second example is the problem of the double diffraction of a plane wave on a pair of skewed edges, formed by two plates placed with a relative angle of $5^{\circ}$, as shown in Fig. 7. As a 


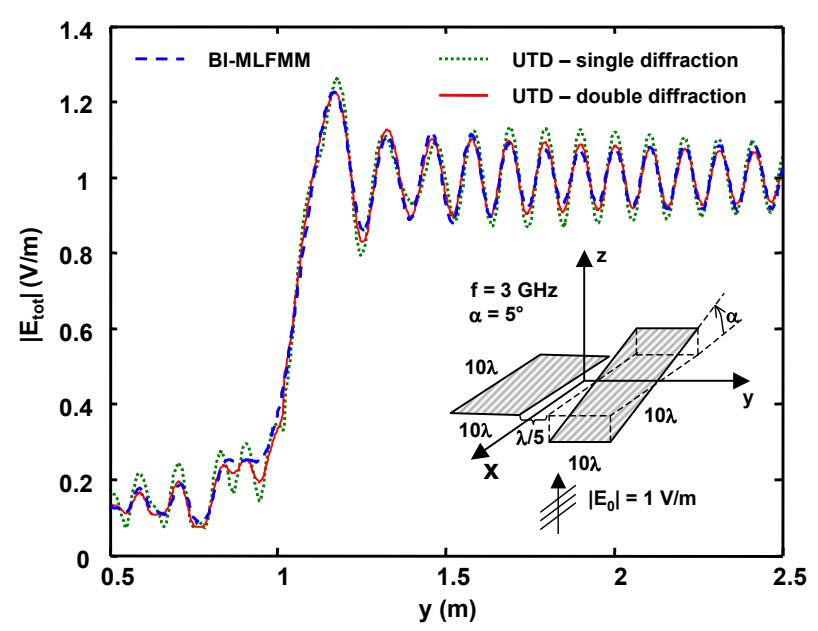

Fig. 7. Double diffraction of plane wave on pair of skewed edges.

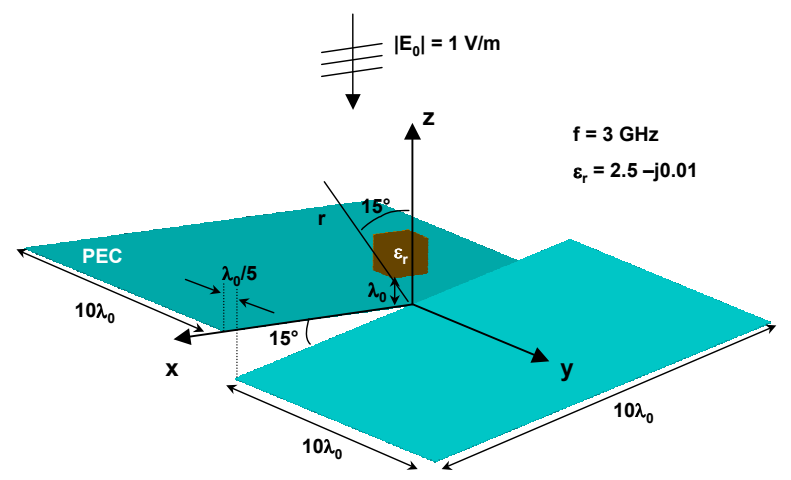

Fig. 8. Dielectric cube over pair of skewed edges.

reference, the full BI solution is used and the ray optical solution was achieved with the hybrid method by activating only the UTD part. In the same figure, the electric near-field distribution along the $y$-axis and $5^{\circ}$ of the z-axis is shown, for a distance of $0.3 \mathrm{~m}$ from the $\mathrm{y}$-axis. This direction corresponds to the Keller cone of the right edge. It can be seen, that considering double diffracted field contributions on the pair of edges, the UTD result shows excellent agreement compared to the reference solution.

Next example is shown in Fig. 8. It consists of a dielectric cube placed over a pair of edges with relative angle of $15^{\circ}$. The dielectric constant of the cube was $\varepsilon_{r}=2.5-j 0.01$ and the frequency $f=3 \mathrm{GHz}$. The problem was excited by a -z-traveling plane wave. In this case, the electric field is observed in a direction along the $y$-axis and $15^{\circ}$ of the $z$-axis for a distance of $r=0.3 \mathrm{~m}$. This direction corresponds to the Keller cone of the right edge of the pair. That way, the field first diffracted on the left edge and then on the right edge of the pair is observed. In Fig. 9, the electric near-field distribution along this direction can be seen. A significant im-

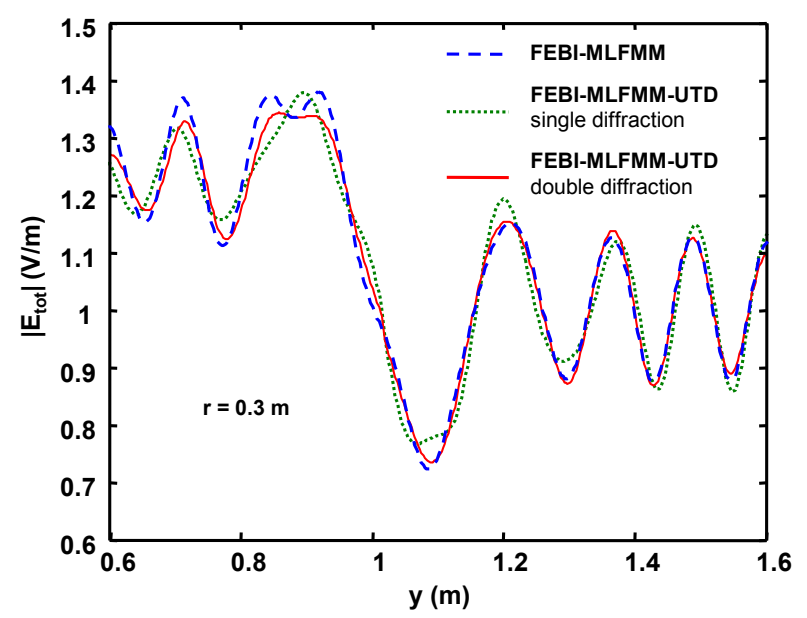

Fig. 9. Electric near-field distribution along direction of observation for configuration shown in Fig. 8.
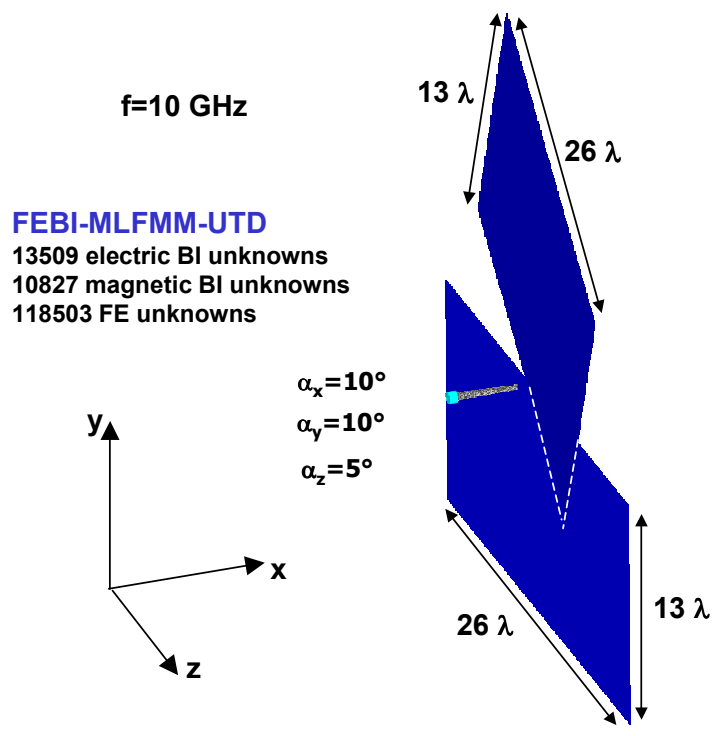

Fig. 10. Dielectric rod antenna radiating in front of skewed flat plates.

provement of the result in the case of considering the double diffracted contribution, compared to the full FEBI reference solution, can be observed.

Last example is a dielectric rod antenna radiating in front of two skewed flat plates, placed as shown in Fig. 10. The operating frequency of the antenna was $10 \mathrm{GHz}$ and a deltagap voltage source was used for excitation. The length of the rod was $3 \lambda_{0}$ with $\varepsilon_{r}=2.5$, where $\lambda_{0}$ is the free-space wavelength, and a metallic mounting was used to hold the rod. The small radius of the rod was $0.24 \lambda_{0}$ and the large radius at the connection point with the metallic mounting was $0.45 \lambda_{0}$. As a reference, the full FEBI solution is used and with the hybrid approach two simulations where performed 


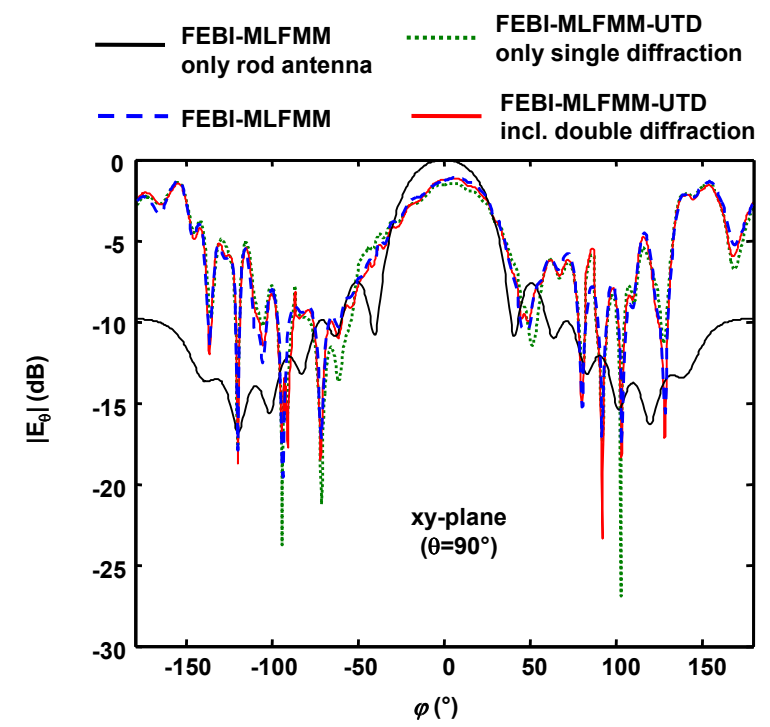

Fig. 11. Radiation pattern in $x y$-plane of the antenna shown in Fig. 10 .

for both, with and without double diffracted contributions on the nearby edges. In Fig. 11, the radiation pattern of the antenna in the $x y$-plane, cutting through the antenna in the middle, is shown. It can be seen, that the hybrid result including double diffraction shows excellent agreement with the reference solution. The simulations are also compared to the radiation pattern of the antenna radiating in free space. The influence of the edges on the radiation of the antenna is obvious. Deformation of the main lobe can be seen, as well as significant increase of the radiation in backward direction. A disturbance of the symmetric radiation can also be observed. The same result can be seen in Fig. 12, where the instantaneous electric field for $t=0 \mathrm{sec}$ is shown in the $x y$-plane. The influence of the edges in the near-field distribution can be clearly seen.

\section{Conclusions}

In this contribution, advances in hybrid FEBI-MLFMMUTD method were presented. In particular, the hybrid technique was extended to double diffracted fields on pairs of straight metallic edges, formulated with the hard and soft scalar diffraction coefficients of UTD. The diffraction points are determined by an iterative three-dimensional parametric realization of the generalized Fermat's principle, allowing treatment of non-coplanar and skewed edges. For the computation of the double diffraction divergence factor, the ray caustic distance of the diffracted field at the second edge is determined by linear interpolation between the radii of curvature in the two principal planes of the incident astigmatic ray tube. Acceleration of the near-field computations in the postprocessing stage of the hybrid method is extended

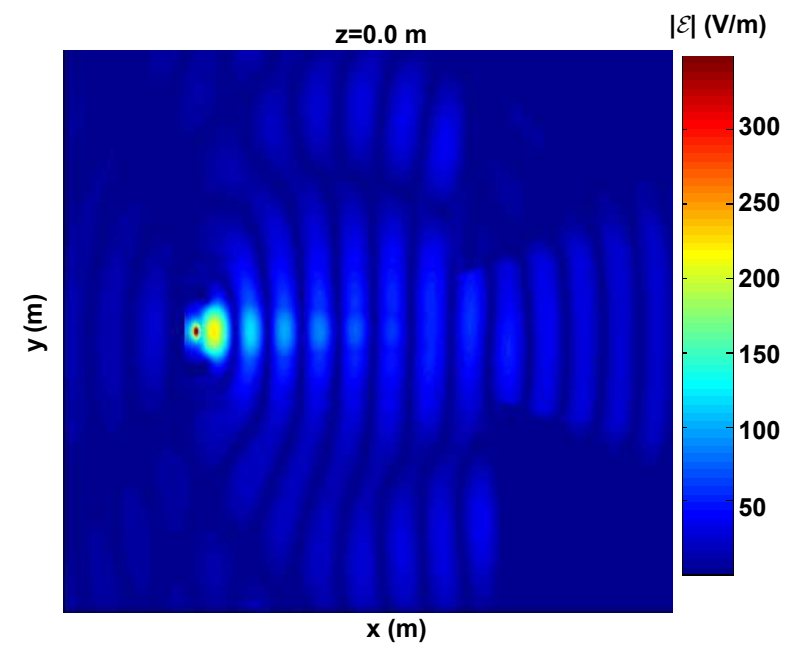

P-MLFMM-UTD on 188426 observation points

Fig. 12. Instantaneous electric field for $t=0 \mathrm{sec}$ in $x y$-plane for the antenna shown in Fig. 10.

in each translation domain to ray optical contributions due to the presence of electrically large objects, according to the hybridization of MLFMM with UTD.

\section{References}

Tzoulis, A. and Eibert, T. F.: A hybrid FEBI-MLFMM-UTD method for numerical solutions of electromagnetic problems including arbitrarily shaped and electrically large objects, IEEE Trans. Antennas Propagat, 53(10), 3358-3366, 2005.

Kouyoumjian, R. and Pathak, P. H.: A uniform geometrical theory of diffraction for an edge in a perfectly conducting surface, Proc. IEEE, 2(11), 1448-1461, 1974.

Tiberio, R., et al.: High-frequency electromagnetic scattering of plane waves from double wedges, IEEE Trans. Antennas Propagat., 37(9), 1172-1180, 1989.

Schneider, M. and Luebbers, R.: A general, uniform double wedge diffraction coefficient, IEEE Trans. Antennas Propagat., 39(1), 8-14, 1991.

Ivrissimtzis, L. P. and Marhefka, R. J.: Double diffraction at a coplanar skewed edge configuration, Radio Sci., 26(4), 821-830, 1991.

Capolino, F., et al.: Double diffraction at a pair of coplanar skew edges, IEEE Trans. Antennas Propagat., 45(8), 1219-1226, 1997.

Albani, M.: A uniform double diffraction coefficient for a pair of wedges in arbitrary configuration, IEEE Trans. Antennas Propagat., 53(2), 702-710, 2005.

Tzoulis, A. and Eibert, T. F.: Efficient electromagnetic near-field computation by the multilevel fast multipole method employing mixed near-field/far-field translations, IEEE Antennas Wireless Propag. Lett., 4, 449-452, 2005. 